\title{
Knowledge Mediation: A Procedure for the Cooperative Construction of Domain Ontologies
}

\author{
Felix-Robinson Aschoff ${ }^{1}$ and Franz Schmalhofer ${ }^{2}$ and Ludger van Elst ${ }^{3}$
}

\begin{abstract}
In order to enable knowledge sharing and reuse among software entities, artificial intelligence researchers have proposed to develop 'ontologies' as the explicit formal specifications of conceptualizations. These ontologies were normally designed by knowledge engineers who laid down the basic categories and relations for a certain domain. However, in any practical setting there will be conflicting interests which pertain to different conceptualizations of which a knowledge engineer will usually not be aware of. For this reason, we propose a three-phased ontology construction procedure in which the knowledge engineer mediates between the differing conceptions experts or users may hold about a knowledge domain. This procedure is described in detail in this paper and subsequently empirically demonstrated and evaluated in a study with 28 participants. The evaluation reveals convincing advantages of the proposed knowledge mediation procedure. We conclude that an ontology construction process is not only an engineering task but more importantly also a social process where the relevant parties for example of a work place need to be involved before successful and durable solutions can be found.
\end{abstract}

Keywords:

Ontology Engineering, Collaboration, Empirical Evaluation

\section{INTRODUCTION}

The field of Artificial Intelligence (AI) has borrowed the term 'ontology' from philosophy, where in its more specific sense it refers to that part of metaphysics that specifies the most fundamental categories of existence, the elementary substances or structures out of which the world is made [1].

In AI an 'ontology' was proposed as an 'explicit specification of a conceptualization' to enable the sharing and reuse of knowledge among software entities [13]. These ontologies were usually designed by knowledge engineers who came to an agreement about the fundamental categories and relations for a certain domain. The ontology was mainly conceived as a

\footnotetext{
${ }^{1}$ Department of Psychology, University of Heidelberg, Germany, Felix-Robinson.Aschoff@urz.uni-heidelberg.de

2 Institute of Cognitive Science, University of Osnabrueck, Germany, Franz.Schmalhofer@uos.de

${ }^{3}$ German Research Center for Artificial Intelligence (DFKI), Kaiserslautern, Germany, elst@dfki.uni-kl.de
}

reference frame for the communication among software agents. These programmable agents were said to commit to an ontology, if their observable actions were consistent with ontological definitions [13]. The acceptance of an ontology by human users, however, has not been a central issue of ontology engineering.

The growing use of ontologies for a wider range of application areas, including Knowledge Management (KM), has shifted the focus to the interaction between human users and ontology-based information systems. For applications like Organizational Memories (e.g. [7]) it is not sufficient that ontologies are only shared conceptualizations among knowledge engineers and domain experts. An essential factor for the success of such KM solutions is its regular usage in the respective work place. This requires the Organizational Memories' ontology to be accepted and understood by endusers and, furthermore, the end-user should be conceived as a valuable source for ontology construction and maintenance.

To establish an ontological commitment in heterogeneous user groups with different perspectives and information needs imposes new challenges for the ontology engineering field. Software agents are programmable entities which exclusively act upon explicated knowledge, i.e. formalized rules and patterns. Human behavior, on the other side, is also governed by implicit or procedural knowledge [17] which is often a result of past working experience. Previous joint activities created a common ground [4] which serves as a base for mutual understanding and coordinated action. From this perspective, an ontology construction process can be conceived in two ways: First as a possibility to create an ontology which reflects this common ground among users and, secondly, as an important means to create this common ground for further ontology-supported collaboration.

Up to now, there are few detailed proposals for the cooperative construction of ontologies in (distributed) groups of human actors (cf. [9]). Existing approaches, like [5][8][21], focus on web-based tools to support the communication between ontology designers. [10] offers comprehensive knowledge acquisition guidelines for ontology construction in expert groups and [22] enables experts to create personal ontologies which can be compared to each other. The process of integrating different viewpoints into one consensual ontology, however, has hardly been investigated yet. The problem of how to establish a consensus and a shared conceptualization, especially when dealing with contradictory knowledge and conflicting interests (e.g. in case of a company 
fusion), has not been dealt with. Furthermore, the role of a human mediator, who supports ontology construction and consensus finding, has not been explored.

We propose a detailed three-phased ontology construction procedure which is especially conceived for a tool-supported development of ontologies in groups of human experts or direct or indirect ontology end-users. Since this procedure is derived from conflict mediation approaches [14] it is especially suitable for heterogeneous groups with possibly conflicting needs and contradictory viewpoints concerning the domain of interest.

This knowledge mediation procedure is evaluated in an experimental study. Since current ontology engineering approaches mostly use discussions to debate about ontological structures (e.g. [21] [22]) we compared the mediation procedure to a condition where an unassisted discussion took place. All trials of our experiment were conducted as three-point videoconference sessions to evaluate the viability of the proposed procedure for ontology construction in distributed user groups over inter- or intranets.

\section{THE KNOWLEDGE MEDIATION PROCEDURE}

The knowledge mediation procedure consists of three main phases. In the generation phase users are jointly brainstorming about relevant concept and instances of the knowledge domain to outline the content of the ontology. During the explication phase each user independently works out a taxonomy by adding definitions and relations to the collected concepts. In the integration phase the knowledge mediator supports the users to integrate their proposed taxonomies into a shared conceptualization. Such a combination of construction and integration phases have already previously been shown to be useful for knowledge engineering tasks [19]. We will exemplify this process for the case of an ontology construction in the research field of Cognitive Science.

\subsection{Generation}

Aim of this phase is to generate a shared list of terms which serves as starting point for the definition of ontological classes. All participants of the construction process are asked to brainstorm about relevant concepts of the knowledge domain. This phase serves to activate the users' knowledge about the domain and is meant to be a first exchange of different perspectives or priorities. Participants are asked to freely generate concepts without any discussion or evaluation by others. Brainstorming guidelines for group sessions as well as supporting software tools can be found at http://www.brainstorming.co.uk. The knowledge mediator makes sure that the brainstorming rules are followed and records the named terms on a white board or a shared application. If a requirement specification document has been set up, she or he takes care that the demands which arise from this document for the ontology are considered during this phase. In case of an ontology for the Cognitive Science research field, the terms named by a user could for example be: Philosophy, Artificial Intelligence, Computer-Metaphor of the Human Mind, Cognitive Modelling, Neuroscience etc.
Top-down, bottom-up, middle-out

The generation of terms can either be done top-down, starting with the most general concepts of a domain, or bottom-up, starting with concrete instances. In accordance with [23] we would recommend a middle-out approach. During the generation phase participants should neither try to think of most general concepts nor should they try to collect all concrete instances which might be relevant. They should rather name those terms which "naturally" occur to them, when they reflect about the domain and their everyday work. This should produce terms which Rosch calls basic level categories [18]. These categories are essential for everyday dealing with the world and are most often used in communication with others. According to Rosch, this is the level at which most of a person's knowledge is organized. This level of abstraction should be a good starting point to model the more abstract and more concrete levels of the ontology later on. It should also result in fewer revisions than a top-down or bottom-up approach.

Support from automatic thesaurus generation tools

Since the participants of the construction process may not have all the relevant knowledge or may not be able to completely recall it during the session, the generation phase should be complemented by automatic thesaurus generation tools (e.g. [2]). These tools can automatically extract key terms from text documents which cover content areas of the domain.

\subsection{Explication}

In this phase, each participant of the mediation process explicates a proposal in form of an ad-hoc taxonomy based on the list of collected terms. This taxonomy should contain the classes of the ontology, their informal descriptions as well as relations among classes. Participants may also propose axioms which they think should be included into the ontology. These proposals are worked out independently without any interference from other participants. Each participant receives time to set up his or her personal structure of the knowledge domain and to reflect about her perspectives, interests and information needs. This phase results in one proposed taxonomy from each user.

\section{Establishing priorities}

Participants should also set priorities during this phase. They should indicate which classes or relations they judge to be especially crucial for their work and which are of lower relevance (e.g. on a scale from 1 to 3 ).

\section{Support from Ontology Mining Techniques}

This phase should also be complemented by results from technical tool support like ontology mining techniques from texts (e.g. [15]). The resulting ontologies from these techniques have the advantage that they do not represent the interests and viewpoints of any participant. Thus, they may serve as neutral instance and can be helpful to reach an agreement during the phase of integration. The selection of these texts can also be carried out in a mediation process. Every participant should be asked to make proposals and a final agreement can be reached by using the techniques described in Section 2.3. The resulting 
text corpora should cover the domain in a balanced and comprehensive way.

\subsection{Integration}

During this phase, the integration of the proposals takes place by means of negotiation and with support of a mediator. We used approaches and techniques from the field of conflict mediation [14] and adapted them to the requirements of an ontology construction process.

In our approach the knowledge engineer takes the role of a knowledge mediator. He has expertise in engineering of ontologies, including the development of sound taxonomies, and ensures that the principles of ontology design (cf. [12][13]) are considered during the construction process. However, he usually does not interfere with the content of the ontology. Hence, he can act as a neutral person who can balance between the different interests and knowledge needs of ontology users. It is his tasks to foster a fair and even negotiation process that results in an ontology which represents a shared understanding of the knowledge domain. There are basic rules for a conflict mediator which we believe also apply for knowledge mediators who support the construction of an ontology:

1) The mediator is responsible for the process of communication, not for the content or the result.

2) The mediator does not come to decisions (or definitions), the participants do.

3) The mediator should remain neutral, considering the interests of all parties.

\section{Perspective Taking}

After the proposals are published for all participants the mediator starts with the negotiation. Perspective taking is a technique from conflict mediation where one party views the world from the perspective of another party. To reach a simple perspective taking effect the mediator asks the participants to introduce the proposal of another participant, to describe it and to outline main differences as well as similarities to her own proposal. The author of the proposal may add additional explanations afterwards.

\section{How to start?}

After this introductory phase the actual negotiation begins. In conflict mediation there are two options: To start with those aspects which are highly controversial or to start with those aspects which can easily be settled. In case of ontology construction, we would recommend to start with those aspects which are of central importance to the ontology (normally the top-level categories) and to reach an agreement for the controversial aspects as early as possible. A settlement of controversial viewpoints at an advanced stage of the negotiation might entail a considerable amount of revisions. Our empirical study, however, showed that if participants start to move in a circle the communication process can sometimes be fostered by changing to aspects which can be settled more easily.
Techniques for the mediator

The following techniques can be used by the mediator to support the process of negotiation:

\section{Balancing}

The mediator should ensure that all participants of the negotiation get an equal chance to express their arguments and ideas. This can e.g. mean to encourage more passive persons to explain their reasoning and to secure that they are not interrupted.

\section{Summarizing:}

The mediator can summarize the state of the negotiation and can preserve already reached agreements. This structures the communication process and helps participants to remain focussed on relevant aspects. Example: "Is it correct that we have not yet reached a joint definition of the class "Cognitive Science' but do agree about its subclasses?"

\section{Messages:}

The mediator should encourage participants of the negotiation to use "I Messages" when arguing about ontological definitions. This means to explain a point of view from a personal perspective, rather than to make apodictic statements. Example: "I would consider 'Cognitive Neuroscience' to be a subclass of Cognitive Science, because....." instead of: "Cognitive Neuroscience obviously is a subclass of Cognitive Science. Therefore, it has to be included into the Ontology."

\section{Paraphrasing}

If experts disagree heavily on crucial concepts, the mediator can try the technique of paraphrasing. He asks an expert to repeat the viewpoint of another expert in his own words. This form of perspective-taking can help to gain an understanding for the standpoints of the others and to come to an integrative definition in a more constructive way.

\section{Useful questions:}

The mediator can use the following questions to urge participants to explicate their knowledge and explain their reasoning:

- "Could you please explain what are the advantages of your structure / definition?"

- "Why did you use this terminology or these relations in the past?"

- "Where do you see the disadvantages of the conceptualization proposed by the other person?"

- Compromising: "If we agree at this point on the definition or relation, which was suggested by you, could we then agree on the other person's suggestion for this other aspect?"

- Reflection about the negotiation process: "Why do you think is it so difficult to reach an agreement about this aspect?" 
- Other participants could be asked to explain their preferences concerning the conflict between two persons.

\section{Neutral Knowledge Sources}

The mediator can refer to the neutral knowledge sources which were described in section 2.2. An ontology which was mined from a corpus of texts is not in the same way shaped by personal interests like the proposed taxonomies. These "neutral" ontologies may serve as orientation to settle disagreements among the participants. Further sources which should be considered during construction are existing ontologies. They can be taken for orientation when design decisions have to be made or may be reused as part of the envisaged ontology.

\section{Analysis of Disagreement}

An analysis of occurring disagreements can help the mediator to understand the reasons for a confrontation. Possible disagreements, which are adapted from [20], are shown in Table 1 .

Table 1. Possible disagreements during the integration phase

\begin{tabular}{|c|c|c|c|}
\hline & \multicolumn{2}{|c|}{ term } \\
\hline & & same & different \\
\hline \multirow{2}{*}{ 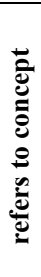 } & $\begin{array}{l}\stackrel{\Xi}{\Xi} \\
\text { J }\end{array}$ & $\begin{array}{l}\text { consensus } \\
\text { participants use terms and } \\
\text { concepts in the same way }\end{array}$ & $\begin{array}{l}\text { correspondence } \\
\text { participants use different } \\
\text { terms for the same concepts }\end{array}$ \\
\hline & 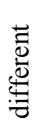 & $\begin{array}{l}\text { conflict } \\
\text { experts use same terms } \\
\text { for different concepts }\end{array}$ & $\begin{array}{c}\text { contrast } \\
\text { experts differ in terms and } \\
\text { concepts }\end{array}$ \\
\hline
\end{tabular}

In case of a consensus, two parties use the same term and refer to the same concept. In case of a correspondence, the participants use different terms but mean the same thing. If, for example, two universities with cognitive science programmes work out an ontology, the fields of "Computer Science" and "Informatics" could cover identical research areas. A conflict occurs, if parties use the same terms but refer to different concepts. Some cognitive science faculties e.g. use the term "Neuroscience" to refer to biological aspects of the human mind whereas other faculties use the same term to refer to psychological aspects. Finally, a contrast occurs when participants' notions of a knowledge domain differ in terms as well as in concepts.

\section{Final agreement}

At the end of the negotiation process, all participants should explicitly declare their agreement to the ontology. Result of the process, however, can also be the decision that a common agreement for a shared conceptualization cannot be reached. In this case, it might be better, if subgroups of the participants define their own ontologies. The procedure will probably show for which composition of subgroups this would be suitable.

\section{Documentation}

A comprehensive documentation of the construction process should be laid down for users of the ontology who did not participate. This documentation should contain: date, participants, represented user groups, proposed taxonomies, main conflicts and how they were solved as well as the final ontology.

\section{EMPIRICAL EVALUATION}

To empirically investigate commonalities and differences between the knowledge mediation approach and a discussion we conducted a controlled laboratory study. Since domain experts or ontology users were not available in sufficient number for an experimental evaluation, we chose students from the Cognitive Science programme at the University of Osnabrueck as participants. Cognitive Science programmes served us as knowledge domain for the ontology construction process. Our participants were experienced in this domain since all students were at least in the second semester of the programme and had already attended classes in most of the main areas the experimental material referred to.

Our experimental scenario was designed to resemble a realistic scenario where two parties with possibly incomplete and contradictory knowledge structures have to construct a joint ontological taxonomy; e.g. in case of a company fusion. We asked our participants to imagine that they would represent a university with a cognitive science programme. Two students at a time were handed out two different programmes. This pair then had to negotiate about a commonly agreed programme structure. One group of students was supported by a mediator and conducted a simplified version of the knowledge mediation procedure whereas the other group negotiated about the programme in an unassisted discussion. After the negotiation, participants filled out a questionnaire and completed a sortingtask. To assess whether the discussion and the mediation procedure could also take place among distributed users we conducted all trials of the experiment via videoconference.

\subsection{Experiment}

\section{Participants.}

28 students who were enrolled in the Cognitive Science programme at the University of Osnabrueck participated in the experiment. 9 students were females and 19 males with an average age of 22.1. All students were at least in the second semester of the study programme and received course credits for their participation.

\section{Apparatus.}

All trials of the experiment were conducted on three Personal Computers in three different rooms of the Usability Lab of the University of Osnabrueck. The PCs were equipped with a Microsoft Windows 2000 Professional operating system and a beta version of daViko 2 multipoint video-conferencing software. The PCs were connected over a local area network with a bandwidth of 100 Mbit. These settings in combination with headsets and gooseneck web cameras allowed each participant to see, hear and speak with the mediator and the other participant as well as to use shared desktop applications (see Figure 1). 
Material.

The experimental material was developed to resemble a situation where a group of experts with incomplete and partially contradictory knowledge has to develop a joint ontological structure. We created two simplified cognitive science programmes based on authentic programmes from the internet. Both programmes contained five main areas of the Cognitive Science field, like 'Philosophy', 'Computer Science', 'Cognitive Psychology' etc. Each of these main areas was presented with informal descriptions and comprised three lectures or subareas. 'Philosophy' in one programme, for example, included 'Philosophy of Mind', 'Philosophy of Science' and 'Theory of Consciousness'. Thus, our experimental material was modelled on a common ontological structure, which consists of classes and subclasses. The two structures differed and contradicted each other in a number of aspects. Only one programme, for example, included the field of 'Computational Linguistics' whereas only the other one included 'Artificial Intelligence'. Both programmes contained 'Neuroscience' but the lectures given under this label had a more biological focus in one programme and a more psychological focus in the other programme.

\section{Procedure.}

The 28 participants were randomly matched into pairs of two. These 14 pairs were then assigned to one of the two experimental conditions: mediation or discussion. The two students of a pair were each handed out a different Cognitive Science programme. The experimenter emphasized that participants should advocate the programme structure of "their university" emphatically during the meeting. Then, they were asked to negotiate about a joint programme.

The pairs in the mediation condition were supported by a mediator and conducted a simplified version of the knowledge mediation procedure (automated ontology mining tools were not yet included into this evaluation). During the integration phase, which lasted 5 minutes, the participants generated relevant terms for the joint programme based on their individual programmes. The mediator created a joint list of these terms by typing them into a shared word editor. In the explication phase, which lasted 10 minutes, the participants worked out a proposal for a joint programme. These proposals were created independently in word editors which were published for the other participant and the mediator at the beginning of the integration phase (see Figure 1). During the integration phase the two participants negotiated for 20 minutes about a joint programme with the assistance of the mediator.

The pairs in the discussion condition were asked to negotiate about the joint programme in an unassisted discussion. This discussion lasted for 35 minutes and the possibilities to use shared editor windows were comparable to the mediation condition. To establish a three party interaction in the discussion condition as well, the experimenter took the role of a recorder who would lay down the agreements but would not interfere in any other way.

\section{Recorded Measures.}

The video streams of all negotiations were recorded and analyzed with respect to speaking time and transitions between participants. In addition to this, a qualitative category system was established to analyze the process of interaction.

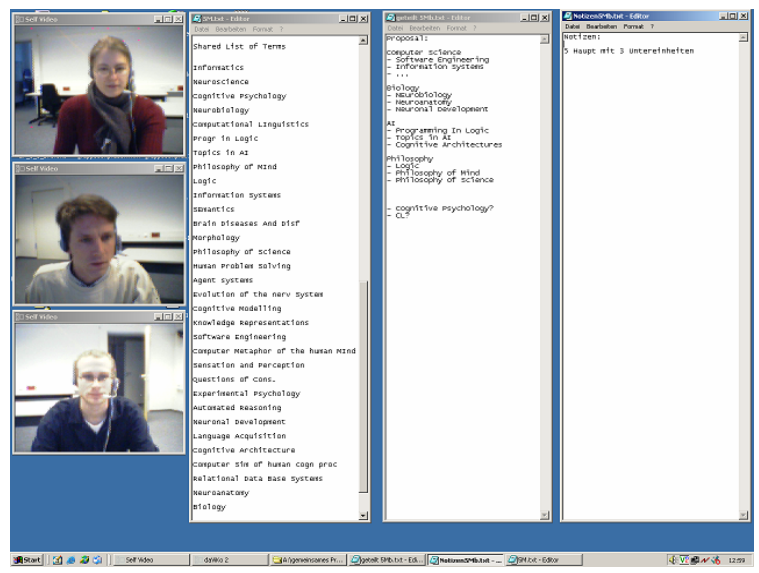

Figure 1. This screenshots shows the mediator as well as a pair of participants. The left word editor shows the shared list of terms and the middle one shows the development of an ad-hoc taxonomy which will be published at the beginning of the integration phase. The right word editor could be used for remarks.

The resulting joint programmes of the pairs were analyzed with respect to the original programmes participants had received at the beginning of the experiment. After the negotiation phase participants filled out a questionnaire. Finally, participants were asked to sort Cognitive Science courses into the joint programme structure. We recorded to what extend this sorting of courses would be identical between the two students of a pair. This should resemble the question if users expect the same content behind a link or an ontological class.

\subsection{Results}

\section{Speaking time:}

The videos were divided into 30 -seconds segments and we analyzed for each segment which speaker dominated the speaking time. Figure 2 shows the mean speaking times for the discussion condition and Figure 3 for the mediation condition. For this analysis the "lead person" was defined to be that participant of a trial who had the larger proportion of speaking time. The "partner" is simply the other person. The "joint talk" category comprises phases of the communication with a high transition rate. During those phases none of the two participants clearly dominated the analyzed 30 -seconds segment. The mediator is not included in this figure since his interventions were usually too short to assign a 30 -second segment to him.

In the mediation condition the "lead person" has a mean speaking time of $36 \%$ and the "partner" a mean speaking time of $23 \%$. This results in a difference of $13 \%$. In the discussion condition, on the other hand, the difference between "lead person" (42\%) and the "partner" (13\%) amounts to $29 \%$. Thus, the surplus of speaking time for the "lead person" is $16 \%$ higher in the discussion condition compared to the mediation condition. 


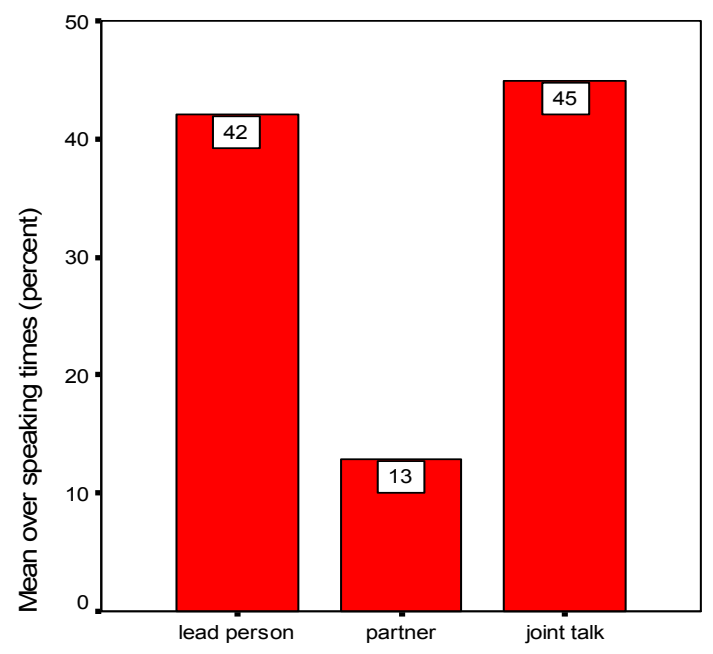

Figure 2. Speaking times for the discussion condition ${ }^{4}$

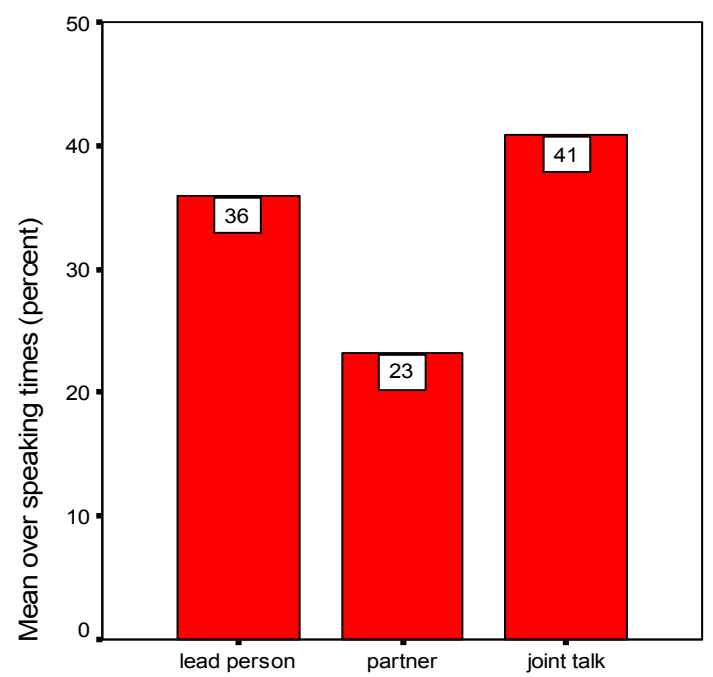

Figure 3. Speaking times for the mediation condition ${ }^{4}$

\section{Transitions:}

Transition frequencies after each 30-second segment were analyzed between the "lead person", the "partner" and the "joint talk" phases. Figure 4 shows the proportional transition frequencies related to all transitions of each trial for the discussion condition and Figure 5 for the mediation condition. The size of the circles represents the share of speaking time reported in Figure 2 and 3. The size of the arrows corresponds to the relative transition frequencies. In addition, the proportional transition frequencies related to all outgoing transitions of the "lead person" as well as the "partner" were calculated. In the discussion condition the "lead person" had $48 \%$ of his or her

${ }^{4}$ These graphs are mainly presented to show the differences between mediation and discussion. The systematic bias which results from the allocation of participants to "lead person" and "partner" can be considered identical in both conditions transitions to herself, $31 \%$ to the "joint talk" and $22 \%$ to the "partner". The "partner" had 5\% to him or herself, $51 \%$ to the "joint talk" and $44 \%$ to the "lead person". In the mediation condition the "lead person" had $37 \%$ to him or herself, $40 \%$ to the "joint talk" and $22 \%$ to the "partner". The "partner" had $30 \%$ to him or herself, $36 \%$ to the "joint talk" and $35 \%$ to the "lead person". Apart from the transitions which are linked to the varying length of speaking time, the transition analysis did not reveal substantial differences between the discussion and the mediation condition.

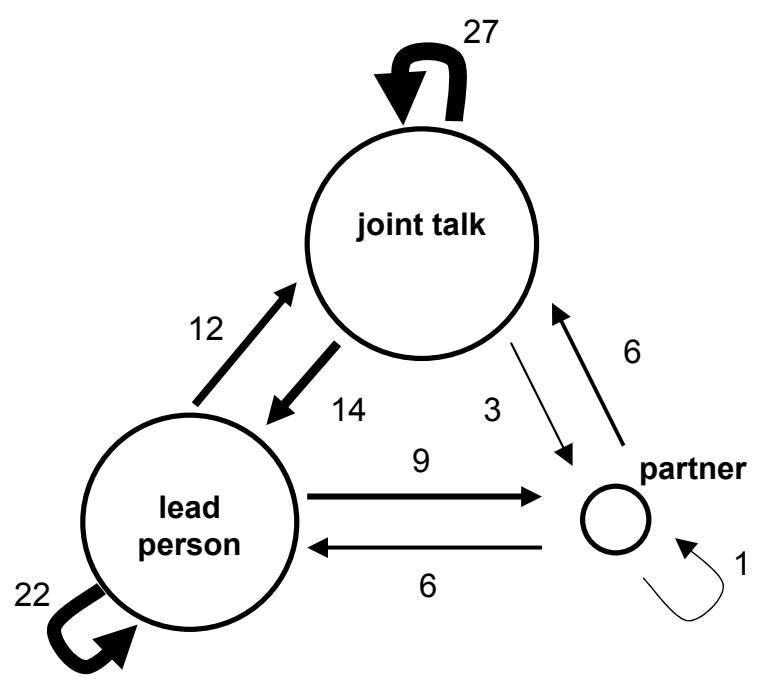

Figure 4. Mean transition frequencies for the discussion (in percent)

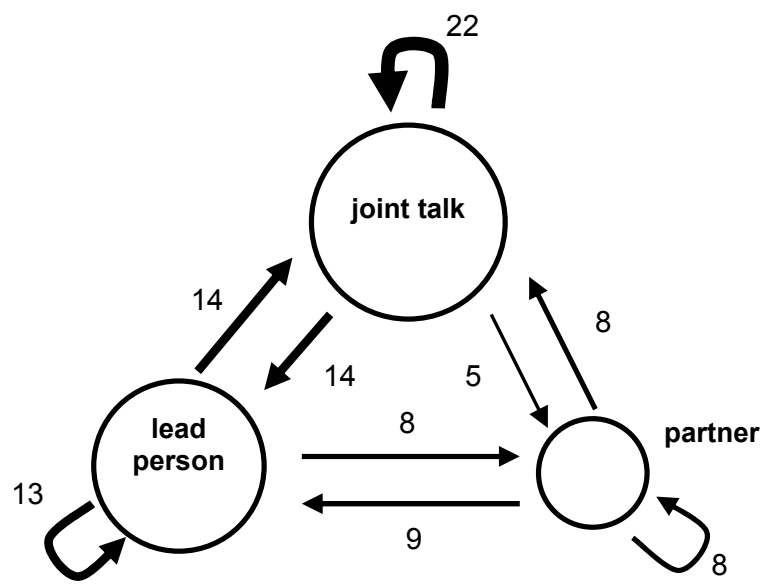

Figure 5. Mean transition frequencies for the mediation (in percent)

\section{Qualitative category system:}

To evaluate the quality of the communication process we analyzed the negotiation between the two students of each trial. The following category system was established: 
a) Comparison of Proposals:

The mediator asked the participants to describe the programme of the other participant and to describe differences to the own programme to reach a simple perspective taking effect.

Example (translated from German): “...We definitely have common aspects in the field of Informatics, I would say.

Concerning the subcategories we would have to decide which are important and which are not and how they fit with my programme. The differences are mainly in the field of Biology, an area I do not have at all, and in Cognitive Psychology..."

\section{b) Description of Programmes:}

One participant describes parts of his programme to the other participant.

Example: “...Well, I have completely different topics at my university. They are: Evolution of the Nervous System, Neuroanatomy and Neurophysiology. (...) What are your topics?" - "Sensation and Perception, Cognitive Modelling and Experimental Psychology..."

\section{c) Consensual Negotiation}

The two participants negotiate about the joint programme in a consensual, agreeing manner.

Example: "...OK, I think Philosophy of Mind is important." "Definitely, yes. And Philosophy of Science?" - "Yes...yes, I think I can agree with that. We can do it that way."

\section{d) Dissensual Negotiation}

The two participants negotiate about the joint programme and disagree with each other or use confronting phrases.

Example: "OK, Artificial Intelligence includes Programming in Logic. Do you agree with that?" - "No. I would prefer to include Programming in Logic in the field of Philosophy..." or

"Now, we come to the only area I basically have to impose on you: Computational Linguistics."

\section{e) Elaborated consensus or dissensus}

Participants do not only exchange statements (like in the example for c) but elaborate on their reasons for their argumentation.

Example: "Well, I think that human language development is a crucial aspect for all approaches which explain cognitive processes. On the one hand, this area is linked to the field of Computer Science and on the other hand, it is linked to Neurobiology and thirdly it is linked to Philosophy, because of the structure of language as such. Because of this I would consider Computational Linguistics as "Integrator" of these different areas. So, I think it should definitely be included into the programme."

Again, we divided the video sequences into 30 -seconds segments and assigned each segment to one of the described categories. The frequencies of the categories are shown in Figure 6 for the discussion condition and in Figure 7 for the mediation condition. The first categories differ due to the different procedures for mediation and for discussion. During mediation a perspective taking or comparison of the two programmes takes places whereas during discussion participants describe their programmes. The phases of consensual negotiations are in both conditions much longer than phases where a dissensus or a confrontation takes places. This probably results from the fact that students were not personally concerned from the outcome of the negotiation. The phases of elaborated consensus or dissensus are short in both conditions compared to entire speaking time, but are considerably higher during mediation $(13 \%)$ in comparison with discussion $(1 \%)$.

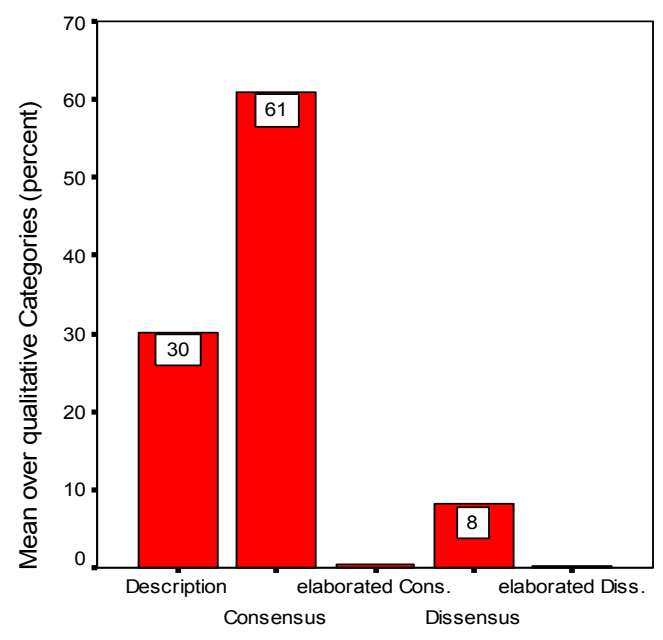

Figure 6. Qualitative categories for the discussion

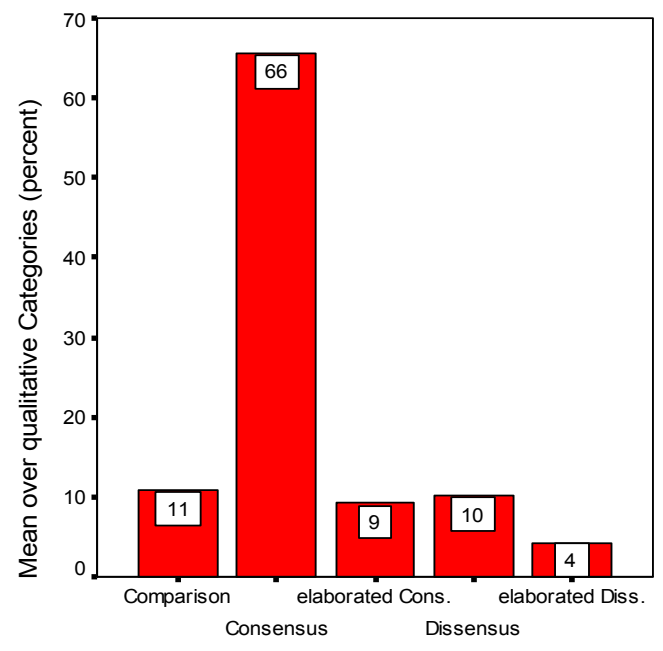

Figure 7. Qualitative categories for the mediation

\section{Evaluation of Joint Programmes:}

We counted how many categories of the joint programme originated from one original programme (A) and how much originated from the other $(\mathrm{B})$. We were interested in the ratio between the programmes which contributed more categories in a trial and the other programme. The programme which contributed more categories was defined as programme 1 . The programme which contributed less was defined as programme 2 . In the discussion condition programme 1 contributed $49 \%$ of the categories and programme $227 \%$ whereas in the mediation condition programme 1 contributed $49 \%$ and programme 2 up 
to $37 \%$. Some categories did appear in both programmes (both) and sometimes participants created new categories which were not included in the original programmes. Categories from both programmes appeared to $11 \%$ for the discussion condition and to $10 \%$ for the mediation condition. Participants created $14 \%$ new categories during discussion and $4 \%$ during mediation. In a total of 14 trials programme A contributed more categories in 7 trials and programme $\mathrm{B}$ contributed more categories in 7 trials, which shows that no programme was preferred by the participants because of its content.

Figure 8 shows the differences between discussion and mediation for the four possible sources. The difference between the contribution from programme 1 and programme 2 is $10 \%$ higher in the discussion condition. On the other hand, participants created $10 \%$ more new categories during discussion.

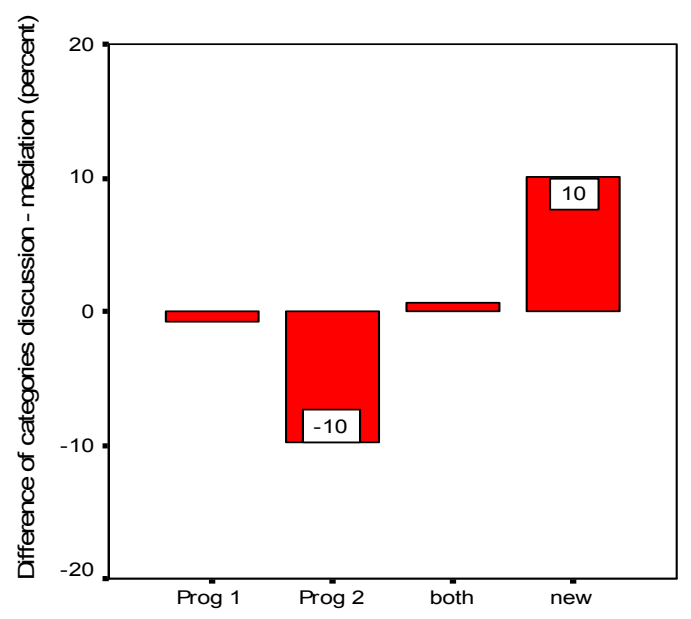

Figure 8. Origin of the joint categories for the discussion

In addition we were interested in the relation between speaking time of a person and how many categories this person contributed to the joint programme. It did not turn out that the person who talks more contributed more categories from her programme than the person who talks less. The lead person contributed only in 6 of 14 trials more categories than the partner ( 3 times during the discussion, 3 times during mediation). The correlation between the proportional speaking time and the proportional number of contributed categories resulted even in a slightly negative correlation of $r=-0.05$.

\section{Questionnaire:}

After the negotiation participants received a questionnaire with 13 questions. 10 questions had to be answered on a 7 point Likert scale, 3 questions were open questions. Participants were asked if they felt that their ideas and suggestions had been considered appropriately during negotiation (particip. considered), if they felt that the other person had been considered appropriately (other considered) and how good the joint programme represented their original programme (represent original). They were also asked to what extend the joint programme covered the content of their original programme (cover original), how good it integrated their original programme (integrate original), and to what extend they thought the joint programme would be suitable for a mutual acknowledgement of course credits (mutual acknowledgement). Further questions referred to the atmosphere of the negotiation (atmosphere), the extendibility of the programme structure for further universities (extendibility), the recognized support through the mediator/recorder (experimenter) and an estimation how good a person who did not participate in the construction process would cope with the joint programme structure (new users). The open question asked if participants liked the experiment and if they had suggestions for improvement of the conducted procedures.

Participant's answers for the questions 1-10 are shown in Figure 9. A number of questions showed a slight tendency in favour of the mediation procedure. This effect, however, did not turn out to be significant in a 10 (Question 1-10) x 2 (mediation, discussion) Analysis of Variance with repeated measure on the first factor. $(\mathrm{F}(1,26)=1,211 \mathrm{p}=.28$ for mediation / discussion).

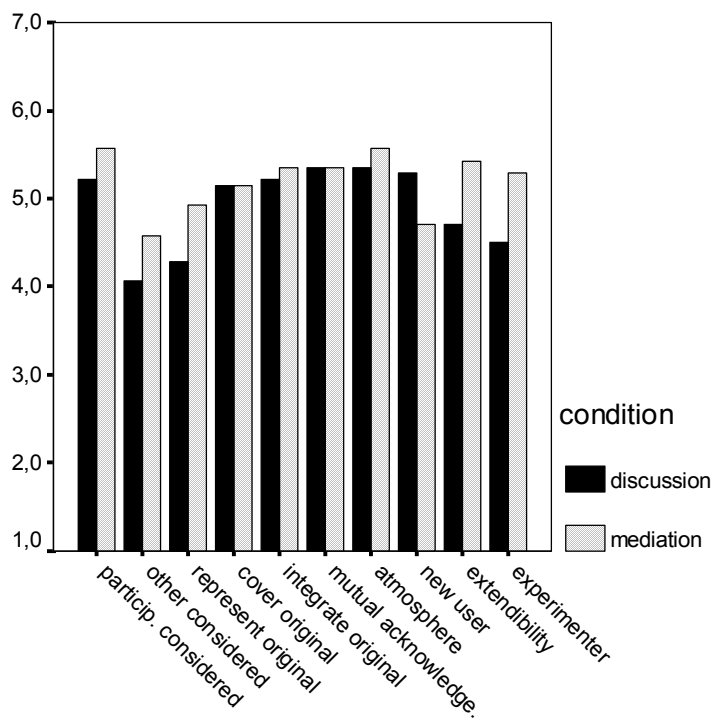

Figure 9. Answers to the questionnaire (7 point Likert scale)

\section{Sorting Task:}

Participants had the task to sort courses into the joint programme. They were asked to decide in what category of the joint programme a course credit was to be acknowledged. Participants received 10 different course credits which had to be assigned to a main area as well as to a subarea. This resulted in a maximum of 20 matches between the participants. We calculated the matches between the two participants of each trial. The pairs of the mediation condition did not have higher matching rates than the pairs in the discussion condition $(\mathrm{M}=16.57 \mathrm{std} .=2.99$ for discussion and $\mathrm{M}=15.29 \mathrm{std} .=3.73$ for mediation).

\subsection{Discussion}

We demonstrated how to create an ontology construction process in a laboratory environment. The participating students were quite dedicated and reported that they enjoyed the experiment, probably because of the interesting and realistic task. The videos showed, that they were less confronting (see also Section 3.2 about the qualitative analysis) than one would expect in a real world situation where more consequences are 
at stake. The students did not always defend the programme of "their university" very strongly. Since mediation is an approach which is especially tailored to conflict situations, we would expect higher differences between discussion and mediation in cases where real interests are at stake (e.g. when two university programmes or companies are merged). Even though our participants were not personally concerned with the outcome of their negotiation, our experiment yielded informative results with respect to these two types of negotiation.

The comparison between discussion and mediation revealed commonalities and certain differences, which indicated an advantage of the mediation procedure in a number of aspects. In general, the mediation turned out to be a more balanced process with respect to the two participants and the two original programmes. The discrepancy between the share of speaking time of one participant compared to the speaking time of the other participant was considerably higher for pairs in the discussion condition than for pairs in the mediation condition. This corresponds to our expected disadvantage for a discussion: This type of negotiation can more easily be dominated by one party.

In the experiment, however, speaking time did not correlate with number of contributed categories. Independent from this, the contributions from the two original programmes are tendentially more balanced in the mediation condition compared to the discussion condition. This tendency corresponds to the speaking times indicating that during discussion one original programme is more likely to dominate the resulting joint programme.

In addition to this, the qualitative analysis revealed important differences between mediation and discussion with respect to the level of communication. During discussion, there is hardly any elaborated argumentation, where participants would explain and reflect upon their statements. Participants during mediation, however, negotiate much more frequently on this elaborated level. The videos indicate that this effect is presumably not only a result of direct mediator intervention. We assume that is also linked to the phases of the procedure. The generation phase and especially the explication phase are likely to foster a reflection about the own perspective of the domain, which can be very fruitful for the ontology construction process. The elaborated negotiation during mediation is a knowledge acquisition process of a much higher quality than the mere exchange of statements during discussion. This difference should be crucial for an ontology construction process which will also rely on the quality of the acquired knowledge.

To sum up, the knowledge mediation procedure fosters a more balanced negotiation process and requests participants to elaborate and reflect their perspectives and viewpoints of a domain. Compared to approaches which draw on discussions among a small number of domain experts and knowledge engineers, the proposed mediation procedure may be more time consuming and requires the involvement of more and possibly distributed persons. Since it will in many cases not be feasible to gather all possible users of an envisaged ontology, we would recommend inviting representatives of respective user groups to the construction process. The knowledge engineer has to deliberate between a sufficiently broad consensus among domain users and a reasonable effort for the construction of the ontology.

We assume that the advantages of our approach are particularly effective in cases of differing or controversial conceptualizations of domain knowledge. Hence, the mediation approach should be especially suitable for the integration of elaborated knowledge structures which users or experts have been holding for a long time. This applies, for example, to scientists who start an (interdisciplinary) collaboration, to experts in case of a company fusion but also to everyday cooperation between employees from different departments, like a research and development, a marketing and a sales department.

Further research has to investigate the effect of the knowledge mediation procedure on a multi-party construction session. Some negative aspects which emerged in our twoparty scenario might even be more predominant if three or more persons are participating. A person's viewpoints and arguments are more likely to be disregarded if more parties try to assert themselves. The mediation approach could prove to be very useful to adequately consider all relevant perspectives. Apart from this, the complex mediation process should be broken down into its basic elements and techniques to reveal their specific effects on the negotiation process. Finally, the connection between a well-balanced negotiation during the conceptualization phase and the success of a resulting ontology-based information system has to be investigated further.

Up to now, there are few well-controlled experimental studies, which evaluate presently used methods for knowledge acquisition and ontology construction (see [3] for a survey). Most guidelines and recommendations arise from field reports which describe methods which seemed to be useful during the development of a specific ontology. While experiments are time consuming and difficult to design, they allow a systematic comparison of existing approaches and techniques. Experimental evaluations should therefore be considered as a crucial means to turn ontology construction from an individual art into a theoretically and empirically founded engineering discipline.

\section{GENERAL DISCUSSION}

We proposed a knowledge mediation procedure for the cooperative construction of ontologies in groups of domain experts or ontology users and showed advantages in an experimental evaluation. Main objective of the procedure is the integration of contradictory knowledge and the establishment of a shared conceptualization as well as a sustainable ontological commitment among human users. The procedure was mainly conceived to reach a consensus about ontological definitions and relations during the conceptualization phase of an ontology life cycle. Additionally, it might also be a fruitful approach for the cooperative creation of a requirement specification document as well as for the integration of contradictory knowledge during the ontology's evolution and maintenance phases.

Even though the knowledge mediation procedure was tailored to the specific needs of human consensus finding it was also conceived as a possible approach for the interaction among software agents. It corresponds to the notion of an ontology society [6] and a society of mind [16] where agents with different rights and obligations interact in a socially coordinated way. As one step towards the long-term goal of frameworks which establish ontological knowledge among 
different actors in (socio-) technological systems, we proposed the possibility of ontology mining techniques as tools to support mediation and assessed the potential of human mediators for consensus finding among collaborating actors.

From the perspective of human-ontology interaction, we oppose the view that ontology engineers should conceptualize the structure of an ontology without integrating the user into the ontology construction process. Many users may not accept the given ontology and may, therefore, decline to interact with an information system. Thus, the construction of an ontology should be conceived as a process in which the cooperating agents negotiate about the rules and structures which apply to their areas of joint activities.

If the ontology construction process is conceived not only as an engineering task but also as a social process its benefits can go beyond the mere development of an ontology:

- Users explicate their implicit knowledge of the domain with benefits for the envisaged ontology as well as for their own expertise.

- Users resp. colleagues benefit from the joint knowledge explication session. It can be a powerful knowledge sharing process and will foster the mutual awareness about who has expertise in what area.

- Users resp. colleagues can jointly define the ontological definitions which form the base for future communication and collaboration. This should lead to a higher commitment and a more sustainable use of the respective systems.

- The knowledge mediation procedure creates a common ground which can establish a fruitful base for coordinated action in the future. Furthermore, it can be an important means to establish a culture of mutual trust and knowledge sharing which often cannot by established by technology alone. As [11] points out: Collaborative systems will not work in a noncollaborative society.

If the process of ontology engineering is also conceived as social process it can be of great value for an organization with respect to the explication of previously unused tacit knowledge, the creation of a culture of knowledge sharing among employees, and the establishment of a corporate identity.

\section{REFERENCES}

[1] Principia Cybernetica Web at http://pespmc1.vub.ac.be/ONTOLI.html

[2] A. Abecker, A. Bernardi, K. Hinkelmann, O. Kühn, M. Sintek, 'Towards a technology for organizational memories'. IEEE Intelligent Systems \& Their Application, 13, 3, (1998).

[3] F.-R. Aschoff \& L. van Elst, Empirical evaluations of Organizational Memory Information Systems: A literature overview, Unpublished DFKI Discussion Paper, 2001. Available: http://serv-4100.dfki.uni-kl.de:8000/ docbase/dokana/WWW/ D00000672.pdf

[4] H. H. Clark, Using Language, Cambridge University Press, Cambridge, 1996.
[5] J. Domingue, Tadzebao and WebOnto: Discussing, browsing, and editing ontologies on the web, In 11th Knowledge Acquisition for Knowledge-Based Systems Workshop, Banff, Canada, 1988.

[6] L. van Elst \& A. Abecker, 'Ontologies for Information Management: Balancing formality, stability, and sharing scope', Expert Systems with Applications, 23, 4, 357-366, (2002).

[7] L. van Elst, A. Abecker, A. Bernardi, A. Lauer, H. Maus, S. Schwarz, An agent-based framework for distributed organizational memories, In: M. Bichler, C. Holtmann, S. Kirn, J. P. Müller, C. Weinhardt (Eds.) Coordination and Agent Technology in Value Networks, Multikonferenz Wirtschaftsinformatik (MKWI-2004), Essen, 181-196, GITO, Berlin, 2004.

[8] A. Farquhar, R. Fikes \& J. Rice, The ontolingua server: A tool for collaborative ontology construction, Knowledge Systems Laboratory, 1996.

[9] M. Fernández-López (Ed.), OntoWeb Deliverable 1.4. A survey on methodologies for developing, maintaining, evaluating and reengineering ontologies, Version 1.0., 2002. Available: http://on to web.aifb.uni-karlsruhe.de/About/Deliverables/ D1.4-v1.0.pdf

[10] M. Fernández-López, A. Gómez-Pérez, J. Pazos-Sierra, 'Building a chemical ontology using METHONTOLOGY and the Ontology Design Environment', IEEE Expert: Special Issue on Uses of Ontologies, 37-46, (1999).

[11] G. Fischer \& J. Ostwald, 'Knowledge management: Problems, promises, realities, and challenges', IEEE Intelligent Systems Journal. Special Issue "Knowledge Management: An Interdisciplinary Approach", 60-72, (2001).

[12] A. Gómez-Pérez, Ontological engineering: A state of the art, Expert Update, 1999.

[13] T. Gruber, 'Towards principles for the design of ontologies used for knowledge sharing', International Journal of HumanComputer Studies, 43, 907-928, (1995).

[14] J. M. Haynes, R. Bastine, G. Link \& A. Mecke, Scheidung ohne Verlierer, Kösel, München, 2002.

[15] A. Maedche \& S. Staab, Mining ontologies from text, In International Conference on Knowledge Engineering and Knowledge Management (EKAW'2000), Juan-Les-Pins, France, 2000.

[16] M. Minsky, The Society of Mind, Simon and Schuster, New York, 1986.

[17] I. Nonaka \& H. Takeuchi, The Knowledge-Creating Company, Oxford University Press, Oxford, 1995.

[18] E. Rosch, C. B. Mervis, W.D. Gray, D.M. Johnson \& P. BoyesBraem, 'Basic objects in natural categories', Cognitive Psychology, 8, 382-349, (1976).

[19] F. Schmalhofer \& B. Tschaitschian, 'Cooperative knowledge evolution: A construction-integration approach to knowledge discovery in medicine', Methods of Information in Medicine, 37, 491-500, (1998).

[20] M. L. G. Shaw \& B. R. Gaines, 'A methodology for recognizing conflict, correspondence, consensus and contrast in a knowledge acquisition system', Knowledge Acquisition, 1, 4, 341-363, (1989).

[21] Y. Sure, M. Erdmann, J. Angele, S. Staab, R. Studer and D. Wenke, OntoEdit: Collaborative ontology engineering for the semantic web, In First International Semantic Web Conference 2002 (ISWC 2002), Sardinia, Italia, 2002.

[22] J. Tennison, \& N.R. Shadbolt, APECKS: A tool to support living ontologies, In B.R. Gaines and M. A. Musen, (Ed.) In 11th Knowledge Acquisition Workshop, Banff, Canada, 1998.

[23] M. Uschold \& M. Gruninger, 'Ontologies: Principles, methods and applications', Knowledge Engineering Review, 11, 2, 93-136, (1996). 